\title{
OPEN Analysis of false-negative rapid diagnostic tests for symptomatic malaria in the Democratic Republic of the Congo
}

\author{
Jonathan B. Parr ${ }^{1,8} \llbracket$, Eddy Kieto ${ }^{2,8}$, Fernandine Phanzu², Paul Mansiangi ${ }^{3}$, \\ Kashamuka Mwandagalirwa ${ }^{3}$, Nono Mvuama ${ }^{3}$, Ange Landela ${ }^{4}$, Joseph Atibu ${ }^{3}$, \\ Solange Umesumbu Efundu ${ }^{5}$, Jean W. Olenga ${ }^{2}$, Kyaw Lay Thwai ${ }^{1}$, Camille E. Morgan ${ }^{6}$, \\ Madeline Denton ${ }^{1}$, Alison Poffley ${ }^{6}$, Jonathan J. Juliano ${ }^{1,6}$, Pomie Mungala ${ }^{2}$, Joris L. Likwela², \\ Eric M. Sompwe ${ }^{5}$, Eric Rogier ${ }^{7}$, Antoinette K. Tshefu ${ }^{3}$, Adrien N'Siala² \& Albert Kalonji ${ }^{2}$
}

The majority of Plasmodium falciparum malaria diagnoses in Africa are made using rapid diagnostic tests (RDTs) that detect histidine-rich protein 2 . Increasing reports of false-negative RDT results due to parasites with deletions of the $p$ fhrp2 and/or $p f h r p 3$ genes ( $p$ fhrp2/3) raise concern about existing malaria diagnostic strategies. We previously identified $p$ fhrp2-negative parasites among asymptomatic children in the Democratic Republic of the Congo (DRC), but their impact on diagnosis of symptomatic malaria is unknown. We performed a cross-sectional study of false-negative RDTs in symptomatic subjects in 2017. Parasites were characterized by microscopy; RDT; pfhrp2/3 genotyping and species-specific PCR assays; a bead-based immunoassay for Plasmodium antigens; and/or wholegenome sequencing. Among 3627 symptomatic subjects, 427 (11.8\%) had RDT-/microscopy + results. Parasites from eight ( $0.2 \%$ ) samples were initially classified as putative $p f h r p 2 / 3$ deletions by PCR, but antigen testing and whole-genome sequencing confirmed the presence of intact genes. $56.8 \%$ of subjects had PCR-confirmed malaria. Non-falciparum co-infection with $P$. falciparum was common (13.2\%). Agreement between PCR and HRP2-based RDTs was satisfactory (Cohen's kappa $=0.66$ ) and superior to microscopy (0.33). Symptomatic malaria due to $p$ fhrp2/3-deleted $P$. falciparum was not observed. Ongoing HRP2-based RDT use is appropriate for the detection of falciparum malaria in the DRC.

Emergence of Plasmodium falciparum strains that evade detection by rapid diagnostic tests (RDTs) threatens progress toward malaria control and elimination in Africa $^{1-3}$. These parasites have deletions involving the histidinerich protein 2 and/or 3 ( $p f h r p 2 / 3$ ) genes, which encode the proteins detected by widely used RDTs throughout Africa $^{4}$. Increasing reports of these parasites in select locations across Africa raise concern about the future of HRP2-based RDTs in the region ${ }^{5-7}$. Recent events in Eritrea, where a high frequency of false-negative RDTs due to these parasites triggered a change in national malaria diagnostic policy, emphasize the need for surveillance and a coordinated response to pfhrp2/3-deleted P. falciparum ${ }^{8,9}$.

We previously performed the first national survey of $p f h r p 2$ deletions and reported a $6.4 \%$ national prevalence of pfhrp2-negative parasites among asymptomatic children in the Democratic Republic of the Congo (DRC) ${ }^{10}$. While this cross-sectional, nationally representative household survey enabled spatial analyses and initial population genetic analyses of $p f h r p 2$-negative parasites in the DRC, it did not sample subjects with symptomatic

${ }^{1}$ Division of Infectious Diseases, Institute for Global Health and Infectious Diseases, University of North Carolina, 130 Mason Farm Rd, Chapel Hill, NC 27599, USA. 'SANRU Asbl (Sante Rurale/Global Fund), Kinshasa, Democratic Republic of the Congo. ${ }^{3}$ University of Kinshasa School of Public Health, Kinshasa, Democratic Republic of Congo. ${ }^{4}$ Institut National Pour La Recherche Biomedicale, Kinshasa, Democratic Republic of the Congo. ${ }^{5}$ Programme National de La Lutte Contre Le Paludisme, Kinshasa, Democratic Republic of Congo. ${ }^{6}$ Department of Epidemiology, Gillings School of Global Public Health, University of North Carolina, Chapel Hill, NC 27599, USA. ${ }^{7}$ Malaria Branch, Division of Parasitic Diseases and Malaria, Centers for Disease Control and Prevention, Atlanta, GA 30033, USA. ${ }^{8}$ These authors contributed equally: Jonathan B. Parr and Eddy Kieto. ${ }^{\square}$ email: jonathan_parr@med.unc.edu 
malaria. In order to inform decisions about national malaria diagnostic testing policy, we undertook a crosssectional survey of children and adults presenting to government health facilities in three provinces selected based on the prevalence of $p f h r p 2$-negative parasites in our initial study. Based on results from our prior study of asymptomatic children and a mathematical model of the spread of $p f h r p 2 / 3$-deleted $P$. falciparum ${ }^{6,10}$, we hypothesized that $p$ fhrp $2 / 3$-deleted parasites were responsible for missed clinical cases of falciparum malaria in the DRC and would be more commonly identified in regions with lower transmission intensity.

Studies of $p f h r p 2 / 3$-deleted P. falciparum are difficult due to the challenges of confirming the absence of these genes using conventional approaches ${ }^{11,12}$. These challenges are compounded by inconsistent laboratory methodologies across studies and inherent limitations of $p f h r p 2 / 3$ assays that can suffer from variable performance and cross-reactivity ${ }^{13,14}$. In addition, false-negative RDT results are common throughout Africa and typically caused by factors other than $p f h r p 2 / 3$ deletions, including operator error, lot-to-lot RDT variability, low-density infections below the RDT's limit of detection, and infection by non-falciparum species ${ }^{15,16}$. In order to overcome these challenges, we performed a comprehensive molecular, serological, and genomic evaluation of symptomatic infections to define the causes of false-negative RDTs in the DRC and inform national diagnostic testing policy.

\section{Methods}

Study population. We conducted a cross-sectional study of subjects presenting to hospitals and health centers across three provinces (Kinshasa, Bas-Uele, and Sud-Kivu) with suspected malaria. Cases of suspected malaria were identified according to national guidelines and included those with fever, headache, convulsions, muscle or joint pain, vomiting, and other symptoms. The National Malaria Control Program considers malaria prevalence in these provinces to be urban, hyperendemic, and mesoendemic, respectively ${ }^{17}$. These provinces were selected based on results from our prior survey of asymptomatic children in the nationally representative 2013-2014 DRC Demographic and Health Survey (DHS) and included provinces with both high- (Kinshasa, Sud-Kivu) and low-prevalence (Bas-Uele) of $p f h r p 2$-negative parasites ${ }^{10}$. We previously estimated the weighted prevalence of $p$ fhrp2-negative P. falciparum among asymptomatic children in these provinces: $21.9 \%$ (95\% confidence interval [CI] 13.9, 32.8]) in Kinshasa, 18.3\% (95\% CI 7.1, 39.9) in Sud-Kivu, and 0.2\% (95\% CI 0, 1.8) in Bas-Uele ${ }^{10}$. Two health zones were selected from each province, including one urban and one rural zone per province. Within each health zone, one general reference hospital and two health centers were selected as study sites, yielding six study sites per province and 18 study sites in total. Subjects of all ages presenting with symptoms compatible with malaria were eligible for enrollment.

Study procedures. Informed consent/assent was obtained from all study subjects prior to enrollment. Parental permission and informed consent was obtained from parents or legal guardians for all minors younger than 18 years of age, and assent was obtained from all children and adolescents 7-17 years of age. All subjects received malaria RDT testing and treatment according to DRC national guidelines. Subjects underwent a study questionnaire and finger- or heel-prick whole blood collection for diagnostic testing by RDT and microscopy and DBS collection. RDT testing was performed using the World Health Organization- (WHO-) prequalified, HRP2-based SD BIOLINE Malaria Ag P.f. (05FK50, Alere, Waltham, MA) according to manufacturer instructions. Thick-smear microscopy slides were read in the field, and thin smears fixed and transported to the National AIDS Control (PNLS) reference laboratory for confirmation and determination of parasite density. All thin smears were read by two microscopists, with discrepancies resolved by a third reader. Dried blood spot (DBS) samples (Whatmann 903 Protein Saver cards, GE Healthcare Life Sciences, Marlborough, MA) were allowed to air dry at ambient temperature in the field, and stored in individual ziplock bags with desiccant at $-20{ }^{\circ} \mathrm{C}$ prior to and after shipment to the University of North Carolina at Chapel Hill for further testing. This study was approved by the Ethical Committee of the Kinshasa School of Public Health (approval number ESP/ $\mathrm{CE} / 07 \mathrm{~B} / 2017$ ). Analysis of de-identified samples and data was determined to constitute non-human subjects research by the UNC Institutional Review Board (study number 17-0155). The study was determined to be non-research by the Centers for Disease Control and Prevention Human Subjects office (0900f3eb81bec92c). Experiments were performed in accordance with relevant guidelines and regulations.

Pfhrp2/3 genotyping by PCR. DNA was extracted from DBS samples using Chelex and saponin ${ }^{18}$. All microscopy-positive, RDT-negative samples, in addition to an equal number of microscopy-positive, RDT-positive controls from each province were subjected to quantitative PCR (qPCR) testing targeting the single-copy P. falciparum lactate dehydrogenase $(p f l d h)$ gene $^{19}$. Pfhrp2 and $p f h r p 3$ PCR genotyping was performed as previously described ${ }^{13}$, using conventional single-step pfhrp2/3 PCR assays and a qualitative real-time PCR assay targeting the single-copy P. falciparum beta-tubulin (PfBtubulin) gene (Supplementary File) ${ }^{20-23}$. Only samples with $\geq 40$ parasites/ $\mu \mathrm{L}$ by $\mathrm{qPCR}$ ( $\geq$ tenfold higher concentration than the $p f h r p 2$ and $p f h r p 3$ assays' limits of detection) were subjected to $p f h r p 2$ and $p f h r p 3$ PCR to reduce the risk of misclassification of deletions ${ }^{13}$. Microscopy-positive, RDT-positive controls with $\geq 40$ parasites/ $\mu \mathrm{L}$ by qPCR were randomly selected from the same facility for $p f h r p 2 / 3$ genotyping. Samples were called $p$ fhrp2/3-negative if they had $\geq 40$ parasites/ $\mu \mathrm{L}$ by $p f l d h$ qPCR, their $p f h r p 2$ and/or $p$ fhrp3 PCR assays were negative in duplicate, and they had successful amplification of PfBtubulin during a final confirmatory assay.

Whole-genome sequencing. All $p$ fhrp2/3-negative samples identified during initial testing were further assessed using whole-genome sequencing. DNA from these samples was enriched for $P$. falciparum prior to library prep using selective whole-genome amplification (sWGA) as previously described ${ }^{24}$. In brief, two sWGA reactions were performed in parallel, one using a custom primer set designed in our lab (JP9) and another 
using a primer set designed by Oyola et al. (Probe_10) ${ }^{25}$. sWGA products were pooled in equal volumes and acoustically sheared using a Covaris E220 instrument prior to library preparation using the Kapa HyperPrep kit (Roche Sequencing, Pleasanton, CA). Indexed libraries were pooled and sequenced at the UNC High Throughput Sequencing Facility using the HiSeq 4000 platform (Illumina, San Diego, CA) with 150 bp, paired-end chemistry. Sequence reads were deposited into the Sequence Read Archive (BioSample accession numbers: SAMN16711875-82).

Evaluation for pfhrp2/3 deletions using whole-genome sequencing. Adapter sequences were trimmed from raw, paired sequence reads using trimmomatic and aligned to the $P$. falciparum 3D7 reference genome (PlasmoDB version 13.0) using bwa mem with default parameters ${ }^{26,27}$. Duplicates were marked and mate-pair information corrected using Picard Tool's MarkDuplicates and FixMateInformation functions, respectively ${ }^{28}$. Candidate indels were identified and realigned using GATK's RealignerTargetCreator and IndelAligner functions, respectively ${ }^{29}$. Genome coverage was calculated using bedtool's genomecov function and visualized using ggplot2 in R (R Core Team, Vienna, Austria) ${ }^{30,31}$. Pfhrp2/3 deletions were called by visualization of aligned reads using the Integrative Genomics Viewer (Broad Institute, Cambridge, MA) and assessment of sequencing depth (the final number of reads that aligned to a specific locus) across the $p f h r p 2 / 3$ genes and their flanking regions ${ }^{32}$. Variant calling was performed using the Genome Analysis Toolkit's HaplotypeCaller ${ }^{29}$. Calls were subset to biallelic SNPs. Variant quality score recalibration was used to filter suspected false positives using the MalariaGEN P. falciparum Genetic Crosses Project (release 1.0) and Pf3k datasets (v5.1). The $\mathrm{F}_{\mathrm{ws}}$ statistic describes the relationship between within-host and population parasite heterozygosity and has been used as an index of genome-wide SNP diversity, with lower values correlated with higher multiplicity of infection, and vice versa ${ }^{33} . \mathrm{F}_{\mathrm{ws}}$ values were calculated using the $v c f d o$ package to identify monoclonal infections; samples with $\mathrm{F}_{\mathrm{ws}}>0.95$ were considered monoclonal ${ }^{34}$.

Antigenemia assessment by Luminex. All DBS samples subjected to $p f h r p 2 / 3$ genotyping by PCR were also assayed for the following Plasmodium antigens: Plasmodium genus-specific aldolase (pAldolase) and lactate dehydrogenase (pLDH), as well as $P$. falciparum HRP2 by a bead-based multiplex assay as previously described ${ }^{35}$. Samples were assayed at 1:20 whole-blood concentration after elution from filter paper. Thresholds for antigen positivity for the three targets were determined by assaying 92 blood samples from US resident blood donors without history of international travel and determining the lognormal mean and standard deviation of assay signal from this sample set. The lognormal mean plus three standard deviations of this sample set was used as the antigen positivity threshold (Supplementary Fig. 1).

Non-falciparum PCR assays. We used R to randomly select 1000 samples for PCR-based species identification. DNA from these samples was first subjected to a pan-Plasmodium real-time PCR assay targeting the $18 \mathrm{~S}$ rRNA gene in duplicate ${ }^{36}$. Any sample with at least one positive pan-Plasmodium replicate was subjected to a series of four $18 \mathrm{~S}$ rRNA real-time PCR assays specific to P. falciparum, Plasmodium malariae, Plasmodium ovale, and Plasmodium vivax in duplicate ${ }^{37-39}$. Species calls were only made if at least two total replicates were positive. Samples with only a single positive pan-Plasmodium replicate but negative species-specific assays were called negative. Samples in which both pan-Plasmodium replicates were positive but species-specific assays negative were subjected to a PCR assay specific to the Plasmodium knowlesi Pkr140 gene ${ }^{40}$. PCR primers and reaction conditions are described in the Supplementary File.

Data analysis. We made comparisons using the Kruskal-Wallis Rank Sum or Fisher's exact test for categorical variables and one-way analysis of variance (ANOVA) test or the t-test for normally distributed continuous variables. Prior to statistical testing, normality was assessed by visual inspection of the frequency distributions of the raw data (histograms) for comparisons with sample sizes $\geq 30$ per subgroup, and using the Lilliefors normality test for comparisons involving sample sizes $<30$ per subgroup. Correlation between parasite density calculations was assessed using the Spearman's rank-order correlation coefficient. Agreement between assays was assessed using Cohen's kappa coefficient. Statistical analyses were performed and maps rendered using R software (version 3.6.2 and 3.6.0, respectively; R Core Team, Vienna, Austria, 2019; www.R-project.org) in RStudio (version 1.2.5033; RStudio, PBC, Boston, USA). Lilliefors test was performed using the nortest package. Maps and figures were generated using the ggplot2, sf, ggspatial, and gridExtra packages and finished in Google Slides (Alphabet, Mountain View, USA).

Disclaimer. The findings and conclusions in this report are those of the authors and do not necessarily represent the official position of the CDC.

\section{Results}

Study subjects. We enrolled 3627 subjects with symptoms compatible with malaria during November and December of the 2017 rainy season, distributed across three provinces: 1203 in Bas-Uele, 1248 in Kinshasa, and 1176 in Sud-Kivu (Fig. 1). Study sites included 18 health facilities located in 18 distinct health areas, spanning six health zones (three health areas per health zone). Baseline characteristics of and malaria diagnostic testing results from enrolled subjects are displayed in Table 1 and Supplementary Table 1. Differences in characteristics and initial malaria diagnostic testing results were observed across provinces. The majority of subjects with recorded temperature data were febrile (median temperature 38 [interquartile range 37, 39]). Study sites included facilities with high, medium, and low symptomatic malaria prevalence. RDT-positive malaria, micros- 


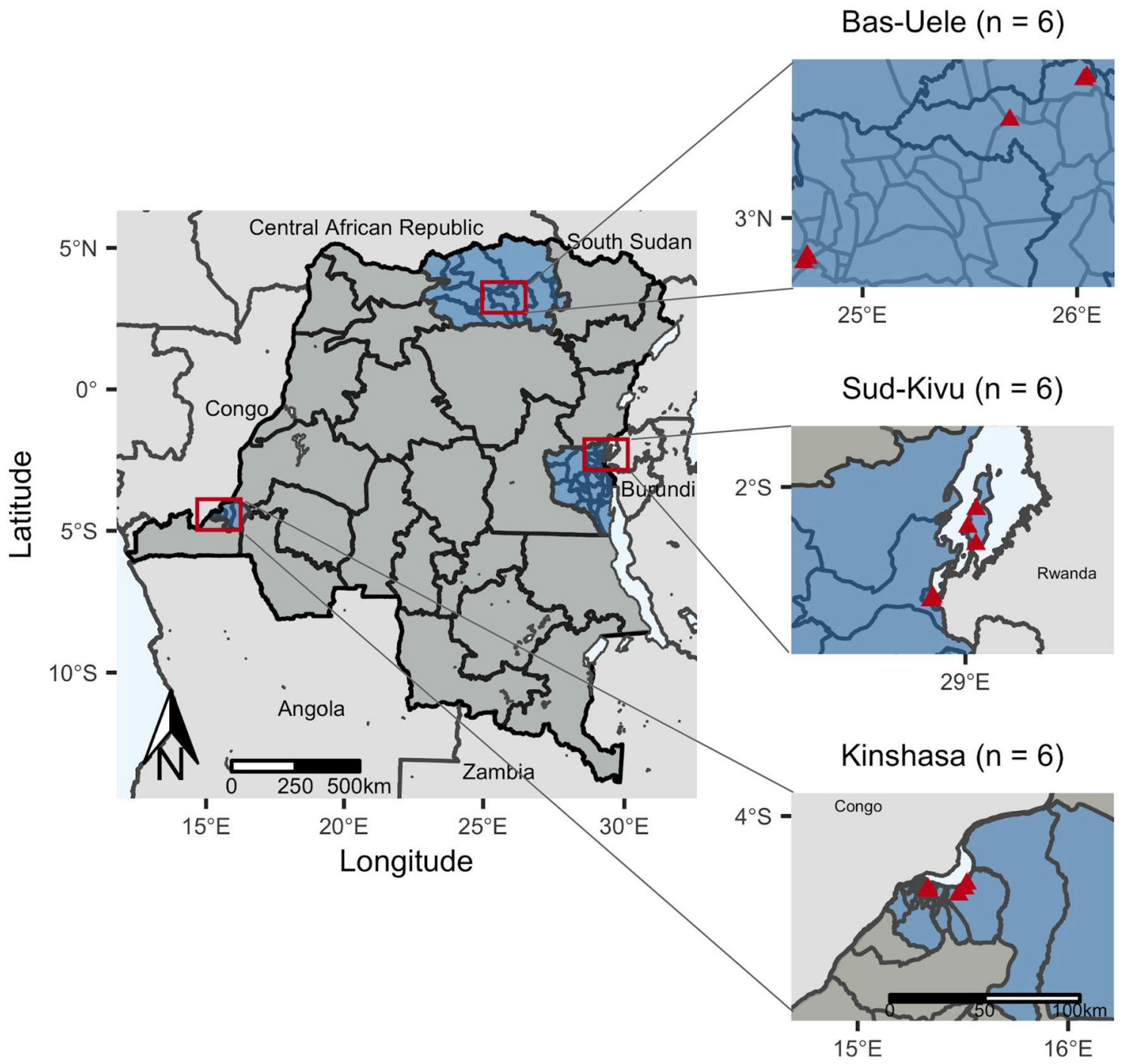

Figure 1. Study sites included health facilities in eighteen health areas (triangles) in six health zones located within three provinces $(n=$ number of facilities per province). Health areas in close proximity have overlapping points. Maps of study sites were rendered in R (R Core Team, Vienna, Austria, 2019, www.R-project.org; version 3.6.0).

copy-positive malaria, and self-reported malaria diagnosis within the past 6 months were highest in Bas-Uele and lowest in Sud-Kivu.

False-negative RDTs by microscopy. Among 3627 subjects tested, 1545 (42.6\%) were RDT-positive and 1397 (38.7\%) were microscopy-positive, with 426 (11.8\%) RDT-negative but microscopy-positive ('false-negative RDT' by microscopy). False-negative RDT results were more likely to occur at low microscopy parasite densities, with geometric means of 959 (geometric standard deviation [SD] 4.2) and 4,675 (geom. SD 6.9) parasites/ $\mu \mathrm{L}$ for RDT- versus RDT + samples, respectively (t-test $\mathrm{p}<0.001$, Fig. 2 ). We observed higher false-negative RDT prevalence in Kinshasa and Sud-Kivu than Bas-Uele, a pattern similar to RDT-/PCR + results we observed in our original $p f h r p 2$ survey in asymptomatic children as part of the 2013-14 DRC DHS ${ }^{10}$. When tested by $p f l d h$ PCR, 368 (86\%) of the 426 RDT-negative/microscopy-positive samples were PCR-negative, a finding consistent with false-positive microscopy calls in this cohort.

Pfhrp2/3 deletion genotyping by PCR. We performed $p f h r p 2 / 3$ genotyping using PCR on a subset of samples, including those collected from all 426 subjects with RDT-/microscopy + results and from 429 RDT + / microscopy + controls selected at random from the same province (Fig. 3). Among the RDT- samples, only 23 had parasite densities sufficient for $p$ fhrp $2 / 3$ deletion genotyping by $p$ fldh $\mathrm{qPCR}(\geq 40 \text { parasites } / \mu \mathrm{L})^{13}$. We further characterized these samples and 74 RDT-positive controls selected from the same facilities $(n=97$ total) using a series of PCR assays for $p f h r p 2$ and $p f h r p 3$, and a final confirmatory PCR assay for PfBtubulin. Eight parasites were PCR-negative for $p f h r p 2$ or $p f h r p 3$ in duplicate despite having parasite densities well above the PCR assays' limits of detection and successful amplification of a second single-copy gene, consistent with $p$ fhrp $2 / 3$ 


\begin{tabular}{|c|c|c|c|c|c|}
\hline & Overall & Bas-Uele & Kinshasa & Sud-Kivu & $\mathbf{p}$ \\
\hline Subjects, $\mathrm{n}$ & 3627 & 1203 & 1248 & 1176 & \\
\hline Health zones, $\mathrm{n}$ & 6 & 2 & 2 & 2 & \\
\hline Health areas, $\mathrm{n}$ & 18 & 6 & 6 & 6 & \\
\hline Age, median years (IQR) & $18(4,32)$ & $17(3,30)$ & $16(4,35)$ & $21(4,33)$ & 0.004 \\
\hline Age strata, n (\%) & & & & & 0.016 \\
\hline$<5$ years & $1025(28.7)$ & $370(31.4)$ & $335(26.8)$ & $320(27.9)$ & \\
\hline $5-14$ years & $579(16.2)$ & $170(14.4)$ & $271(21.7)$ & $138(12.0)$ & \\
\hline $15-24$ years & $639(17.9)$ & $232(19.7)$ & $181(14.5)$ & $226(19.7)$ & \\
\hline $25-34$ years & $513(14.4)$ & $170(14.4)$ & $144(11.5)$ & $199(17.4)$ & \\
\hline $35-44$ years & $344(9.6)$ & $101(8.6)$ & $131(10.5)$ & $112(9.8)$ & \\
\hline $45-54$ years & $249(7.0)$ & $73(6.2)$ & $108(8.7)$ & $68(5.9)$ & \\
\hline 55 years and older & $223(6.2)$ & $62(5.3)$ & $78(6.2)$ & $83(7.2)$ & \\
\hline Female gender, n (\%) & $2130(58.7)$ & $780(64.8)$ & $646(51.8)$ & $704(59.9)$ & $<0.001$ \\
\hline Pregnant, n (\% of women) & $350(16.4)$ & $105(13.5)$ & $26(4.0)$ & $219(31.1)$ & $<0.001$ \\
\hline Slept under bednet the night before, $\mathrm{n}(\%)$ & $2238(79.5)$ & $586(75.9)$ & $902(80.9)$ & $750(80.9)$ & 0.014 \\
\hline Diagnosed with malaria in the last six months, n (\%) & $1556(43.1)$ & $758(63.0)$ & $462(37.6)$ & $336(28.6)$ & $<0.001$ \\
\hline Microscopy-positive, n (\%) & $1397(38.7)$ & $500(41.7)$ & $533(43.0)$ & $364(31.0)$ & $<0.001$ \\
\hline RDT-positive, n (\%) & $1545(42.6)$ & $758(63.0)$ & $380(30.4)$ & $407(34.6)$ & $<0.001$ \\
\hline RDT-negative, microscopy-positive, n (\%) & $426(11.8)$ & $51(4.2)$ & $267(21.5)$ & $108(9.2)$ & $<0.001$ \\
\hline Parasites/ $\mu \mathrm{L}$ by microscopy, geometric mean (geometric SD) & $2877(6.9)$ & $3739(6.5)$ & $1789(7.2)$ & $4001(6.3)$ & $<0.001$ \\
\hline
\end{tabular}

Table 1. Characteristics of enrolled study subjects. Kruskal-Wallis and one-way ANOVA p-values are included for province-wise comparisons. $I Q R$ interquartile range, $R D T$ rapid diagnostic test, $S D$ standard deviation.

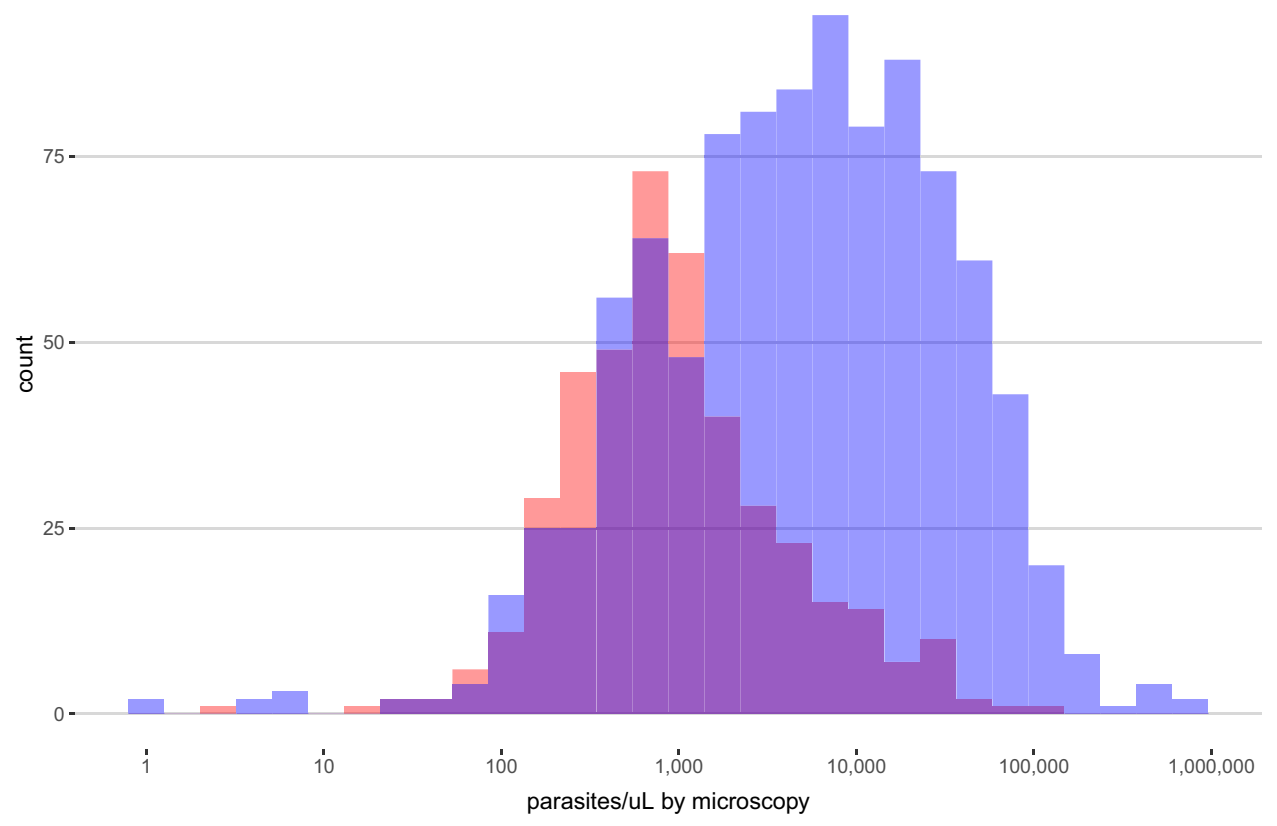

Figure 2. Parasite densities by microscopy among HRP2-based RDT-negative (red) and RDT-positive (blue) isolates. False-negative RDT results were more likely to occur at low microscopy parasite densities, with geometric means of 959 (geometric standard deviation [SD] 4.2) and 4675 (geom. SD 6.9) parasites/ $\mu \mathrm{L}$ for RDTnegative versus RDT-positive samples, respectively ( $\mathrm{t}$-test $\mathrm{p}<0.001)$. $R D T$ rapid diagnostic test. 


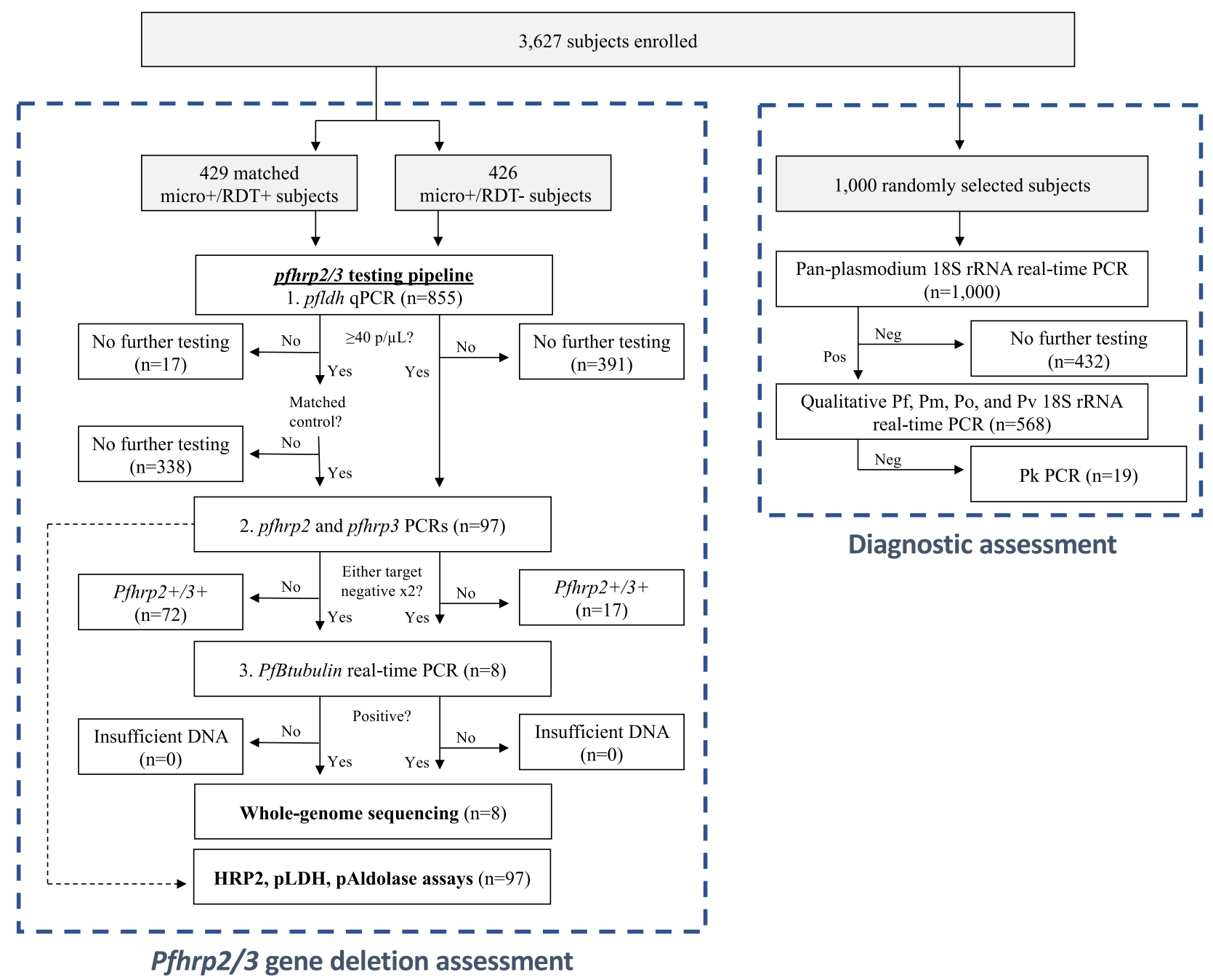

Figure 3. Sample selection for assessment of $p f h r p 2 / 3$ deletions and diagnostic performance. Pf P. falciparum, Pm P. malariae, Po P. ovale, Pv P. vivax, Pk P. knowlesi.

gene deletions using conventional PCR genotyping criteria ${ }^{4,5,41}$. PCR genotyping suggested five deletions among symptomatic RDT-/microscopy + subjects (four pfhrp2-/3- and one pfhrp2-13+) and three among RDT +1 microscopy + subjects (one $p f h r p 2-13+$, one $p f h r p 2+/ 3-$, and one $p f h r p 2-13-$ ) (Supplementary Table 2). Parasite densities for these eight putative $p f h r p 2 / 3$-deleted samples ranged from 84 to 102,700 parasites/ $\mu \mathrm{L}$ by qPCR (median 2929, interquartile range 1314 to 4572 ).

Whole-genome sequencing of candidate pfhrp2/3 deletions. However, whole-genome sequencing (WGS) confirmed that all eight putative $p f h r p 2 / 3$-deleted samples had parasites with intact $p f h r p 2$ and $p f h r p 3$ genes (Fig. 4). All eight samples had at least 5 aligned reads across $>80 \%$ of the genome, with median aligned reads ranging from 66-254 reads/position (Supplementary Fig. 2). Regions of reduced sequencing depth corresponded to differences in the number of histidine repeats compared to the 3D7 reference sequence and did not introduce frame-shift mutations. Mutations in PCR primer binding sites were not observed. $\mathrm{F}_{\mathrm{ws}}$ values suggested monoclonal infection in only 3 (37.5\%) of the 8 samples subjected to whole-genome sequencing (Supplementary Table 3).

Antigenemia assessment using a bead-based immunoassay. Luminex-based serological assessment further confirmed that all eight $p$ fhrp2/3-PCR-negative samples had detectable HRP2 antigenemia, providing confidence that the intact genes observed during whole-genome sequencing encoded functional HRP2 and/ or P. falciparum histidine-rich protein 3 (HRP3) proteins. Background-subtracted mean fluorescence intensity (MFI) thresholds for positivity were 29, 62, and 72 for HRP2, pLDH, and pAldolase, respectively (Supplementary Figs. 1 and 3). We assessed HRP2 antigenemia in all 97 samples that had undergone $p$ fhrp2/3 genotyping by PCR. Comparing RDT-negative versus RDT-positive samples, positivity and background-subtracted mean fluorescence intensity (MFI) was similar between groups for all three antigens tested: HRP2, Plasmodium parasite lactate dehydrogenase ( $\mathrm{pLDH})$, and Plasmodium parasite aldolase (pAldolase) (Table 2). Surprisingly, the 


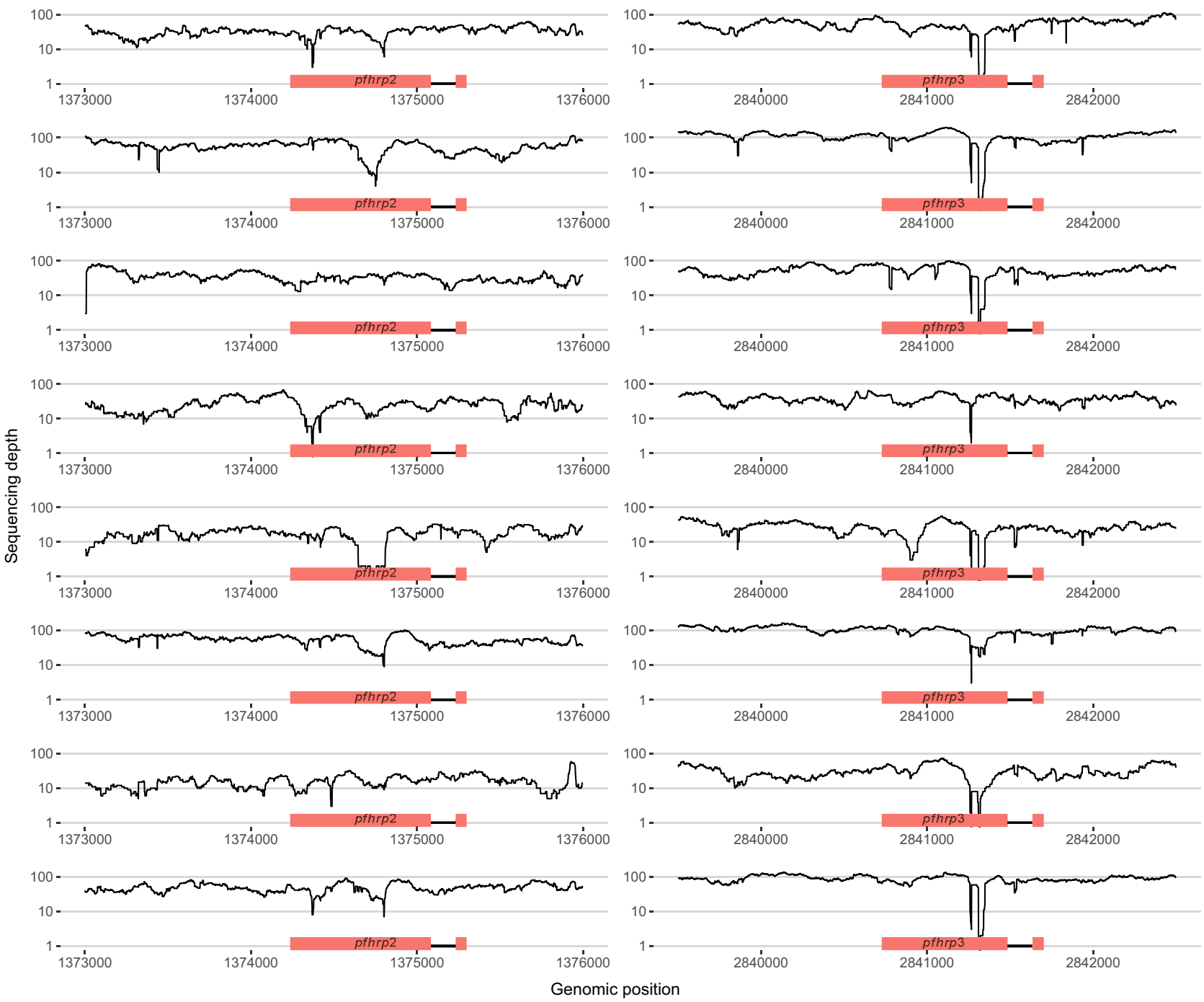

Figure 4. No $p f h r p 2$ or $p f h r p 3$ deletions were identified by whole-genome sequencing. WGS of five $p f h r p 2-/ 3-$, two $p f h r p 2-13+$, and one $p f h r p 2+/ 3$ - parasites originally identified by PCR confirmed intact genes. Each row represents a distinct sample: from SANHRP_01 (top) to SANHRP_08 (bottom). Depth of coverage statistics and sample characteristics are provided in Supplementary Fig. 2 and Supplementary Tables 2-3.

\begin{tabular}{|l|l|l|l|l|}
\hline & Overall & RDT-negative & RDT-positive & p \\
\hline Tested, $n$ & 97 & 23 & 74 & \\
\hline HRP2-positive, $n$ (\%) & $96(99.0)$ & $22(95.7)$ & $74(100.0)$ & 0.24 \\
\hline HRP2, MFI-background geom. mean (geom. SD) & $10,276(2.9)$ & $11,751(1.7)$ & $6,675(7)$ & 0.90 \\
\hline pLDH-positive, $n$ (\%) & $72(74.2)$ & $15(65.2)$ & $57(77.0)$ & 0.28 \\
\hline pLDH, MFI-background geom. mean (geom. SD) & $408(8.4)$ & $440(9)$ & $319(6.5)$ & 0.52 \\
\hline pAldolase-positive, $n$ (\%) & $92(94.8)$ & $21(91.3)$ & $71(95.9)$ & 0.56 \\
\hline pAldolase, MFI-background geom. mean (geom. SD) & $1419(5.5)$ & $1571(5.4)$ & $1022(5.9)$ & 0.46 \\
\hline
\end{tabular}

Table 2. Plasmodium antigen immunoassay results. Frequencies and log-transformed mean values were compared using the Fisher's exact test and Kruskal-Wallis rank sum test, respectively. MFI-background values are visualized in Fig. 5. RDT rapid diagnostic test, $M F I$-background mean fluorescence intensity minus background, $S D$ standard deviation. 
HRP2

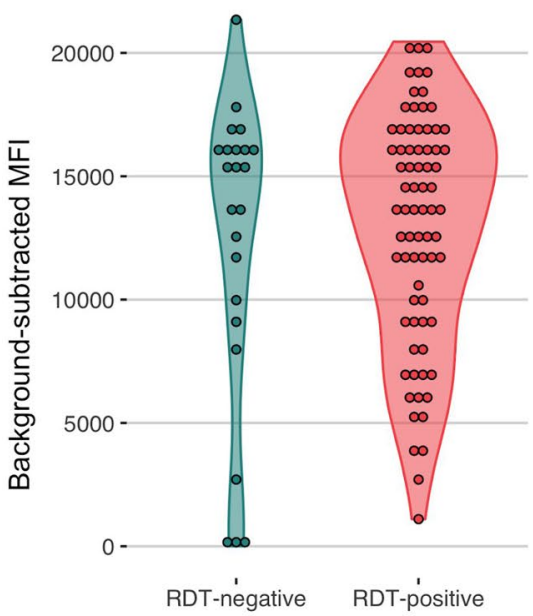

pAldolase

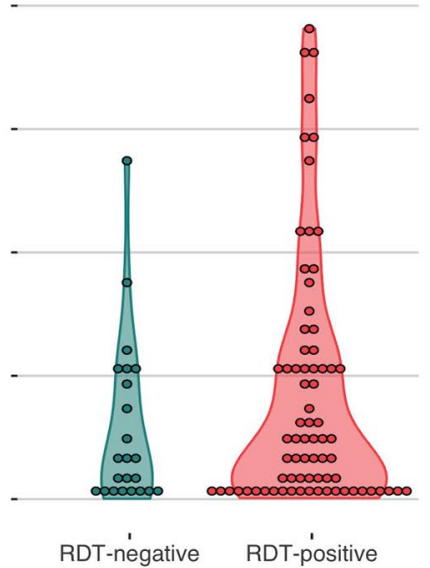

pLDH

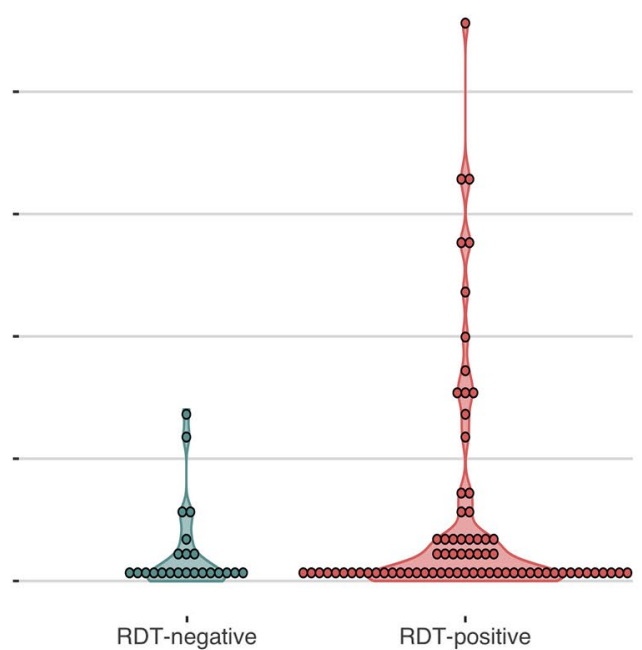

Figure 5. False-negative RDT results occurred in the setting of high HRP2 antigenemia. A Luminex beadbased immunoassay for three parasite antigens confirmed circulating HRP2 antigen in the majority of RDTnegative but PCR-positive/microscopy-positive isolates tested (RDT-negative). RDT-positive controls selected from the same facility are included for comparison. One $\mathrm{pLDH}$ value with background-subtracted $\mathrm{MFI}<0$ is not depicted. Log-transformed plots are provided in Supplementary Fig. 3. HRP2 P. falciparum histidine-rich protein 2, pAldolase Plasmodium aldolase, pLDH Plasmodium lactate dehydrogenase.

\begin{tabular}{|l|l|l|l|l|r|}
\hline & Overall & Bas-Uele & Kinshasa & Sud-Kivu & p \\
\hline $\mathrm{n}$ & 1000 & 328 & 353 & 319 & \\
\hline Plasmodium (any species) PCR-positive, n (\%) & $568(56.8)$ & $280(85.4)$ & $114(32.3)$ & $174(54.5)$ & $<0.001$ \\
\hline P. falciparum PCR-positive, n (\%) & $538(53.8)$ & $268(81.7)$ & $104(29.5)$ & $166(52.0)$ & $<0.001$ \\
\hline Species identification by PCR, n (\%) & & & & & 0.001 \\
\hline P. falciparum only & $463(81.5)$ & $210(75.0)$ & $97(85.1)$ & $156(89.7)$ & \\
\hline P. malariae only & $2(0.4)$ & $2(0.7)$ & $0(0.0)$ & $0(0.0)$ & \\
\hline P. ovale only & $6(1.1)$ & $1(0.4)$ & $4(3.5)$ & $1(0.6)$ & \\
\hline P. vivax only & $2(0.4)$ & $1(0.4)$ & $0(0.0)$ & $1(0.6)$ & \\
\hline Mixed Pf and Pm & $16(2.8)$ & $12(4.3)$ & $1(0.9)$ & $3(1.7)$ & \\
\hline Mixed Pf and Po & $53(9.3)$ & $42(15.0)$ & $5(4.4)$ & $6(3.4)$ & \\
\hline Mixed Pf and Pv & $2(0.4)$ & $1(0.4)$ & $0(0.0)$ & $1(0.6)$ & \\
\hline Mixed Pm and Po & $1(0.2)$ & $1(0.4)$ & $0(0.0)$ & $0(0.0)$ & \\
\hline Mixed Pf, Pm, Po & $4(0.7)$ & $3(1.1)$ & $1(0.9)$ & $0(0.0)$ & \\
\hline Plasmodium positive, species undetermined & $19(3.3)$ & $7(2.5)$ & $6(5.3)$ & $6(3.4)$ & \\
\hline
\end{tabular}

Table 3. Species identification by PCR among subjects with symptomatic malaria. Results of Plasmodium genus and species-specific $18 \mathrm{~S}$ rRNA real-time PCR assays. Kruskal-Wallis p values are included for provincewise comparisons. Pf P. falciparum, Pm P. malariae, Po P. ovale, Pv P. vivax.

majority of all RDT- samples tested had high levels of circulating HRP2 detected by Luminex, suggesting that the negative RDT results were likely due to operator error or RDT failure (Fig. 5, Supplementary Fig. 3).

Non-falciparum malaria. Non-falciparum malaria is expected to cause HRP2-RDT-negative/microscopypositive results and was common in our study cohort. Among 1000 randomly selected samples that underwent species identification using a series of real-time PCR assays (Fig. 3), malaria was confirmed by PCR in 56.8\% of samples, and non-falciparum co-infection with P. falciparum was common (13.2\%, $\mathrm{n}=75$ ) (Table 3). However, only $1.9 \%(\mathrm{n}=11)$ of symptomatic cases were due to non-falciparum infections alone. $P$. ovale was observed in $11.2 \%(n=64)$ of Plasmodium-PCR-positive symptomatic cases. Among the four $(0.8 \%)$ symptomatic cases involving $P$. vivax, half involved $P$. falciparum and all were low density $(<5$ parasites/ $\mu \mathrm{L}$ by semi-quantitative $18 \mathrm{~S}$ rRNA PCR). The majority of symptomatic $P$. malariae infections $(86.9 \%, n=20 / 23)$ occurred as part of mixed infections with P. falciparum (Supplementary Fig. 4). We were unable to determine the species in 19 samples that were positive by the pan-species $18 \mathrm{~S}$ PCR assay in duplicate; all had negative $P$. knowlesi PCR results. 
RDT performance. Assessment of RDT performance versus PCR suggested that false-negative RDT results in our cohort were commonly caused by RDT failure or operator error rather than parasite factors. Among the random subset of 1000 samples that underwent 18S rRNA testing for all species, 134 (24.9\%) of 538 P. falciparum 18S rRNA real-time PCR-positive samples were RDT-negative. RDT performance varied by province, with a larger proportion of RDT-/PCR + results in provinces with higher $P$. falciparum prevalence by $18 \mathrm{~S}$ rRNA PCR: Bas-Uele (19\%), followed by Sud-Kivu (17\%), and finally Kinshasa (5\%) (Supplementary Table 4). Only a small proportion of samples $(3.6 \%, \mathrm{n}=36)$ were $\mathrm{RDT}+/ \mathrm{PCR}-$, a finding not unexpected and suggestive of persistent PfHRP2 antigenemia after recent clearance of parasitemia ${ }^{42}$. When compared to PCR, RDTs were $75 \%$ sensitive and $92 \%$ specific, with good agreement (Cohen's kappa $=0.66$ ). Microscopy was $53 \%$ sensitive and $81 \%$ specific, with fair agreement with PCR (Cohen's kappa $=0.33$ ). Parasite densities as determined by microscopy and $p f l d h$ qPCR had moderate correlation (Spearman correlation coefficient $=0.63, \mathrm{p}<0.001$, Supplementary Fig. 5).

\section{Discussion}

We did not observe symptomatic malaria due to $p f h r p 2$ - or $p f h r p 3$-deleted $P$. falciparum in this large, crosssectional survey across three geographically disparate DRC provinces. The majority of RDT-negative/microscopypositive results occurred in the setting of low or absent parasitemia. This finding implicates parasite densities below the RDT's limit of detection and false-positive microscopy results as the primary causes of RDT-microscopy discordance in the present study. Further assessment of RDT performance using microscopy, genus- and species-specific real-time PCR assays, and Luminex-based antigenemia assessment confirmed that RDT failure and/or user error also caused false-negative RDTs in the present study. However, the overall performance of HRP2-based RDTs was superior to microscopy and in good agreement with PCR.

These findings support continued use of HRP2-based RDTs in the DRC. They also contrast with the results of our prior study of asymptomatic children enrolled in the 2013-2014 DHS. There are several possible explanations for these differences. The present study enrolled symptomatic subjects in order to directly inform policy decisions about malaria case management. This study design could have inhibited our ability to identify $p f h r p 2 / 3-$ deleted parasites. We and others have proposed the hypothesis that parasites with deletions of the $p$ fhrp 2 and/or $p f h r p 3$ genes and their flanking regions may be less fit ${ }^{6,10,43}$, and less likely to cause symptomatic disease. Direct assessment of this hypothesis has not yet been performed in vivo or in vitro, to our knowledge, and is limited by the challenges of confirming deletions in low parasite density infections. However, genetic cross experiments of the 3D7 (wild-type), DD2 ( $p f h r p 2$-deleted), and HB3 ( $p f h r p 3$-deleted) lab strains did not provide definitive evidence of a fitness cost associated with deletion of either gene ${ }^{44,45}$. In addition, reports from Eritrea confirm that $p$ fhrp2/3-deleted parasites can cause symptomatic and sometimes severe disease ${ }^{8}$.

Exhaustive analysis of putative $p f h r p 2 / 3$-deleted parasites was needed to discern the status of both genes. The use of rigorous parasite density thresholds well above the downstream $p f h r p 2 / 3$ PCR assays' limit of detection ${ }^{13}$, confirmation of successful amplification of multiple single-copy genes, and adherence to commonly accepted criteria $^{5}$ reduced the risk of inappropriate $p f h r p 2 / 3$ deletion calls. Only five of 426 (1.2\%) RDT-negative/microscopy-positive samples were identified as putative $p f h r p 2 / 3$ deletions during initial testing. However, we subsequently confirmed HRP2 antigenemia and intact $p f h r p 2$ and $p f h r p 3$ genes in all eight $p f h r p 2 / 3$-PCR-negative samples using highly sensitive antigen detection methods and WGS, respectively. Pfhrp 2/3 sequence variation and resulting changes in the structure of the HRP2 and HRP3 proteins could yield a negative RDT but positive Luminex result, or vice versa, due to differences in the anti-HRP2 monoclonal antibodies employed by both assays. However, previous evaluation of diverse $P$. falciparum strains failed to identify an association between pfhrp $2 / 3$ sequence variation and RDT sensitivity ${ }^{46}$. Taken together, Luminex and WGS results confirmed that the putative $p f h r p 2 / 3$-deleted parasites were misclassified during initial PCR testing.

These findings emphasize the challenges of confirming $p$ fhrp $2 / 3$ gene deletions and support the argument that a portion of $p f h r p 2 / 3$ deletion calls in our original study of asymptomatic children in the DRC resulted from experimental artifact ${ }^{10,11}$. Even complex laboratory workflows conducted in accordance with commonly used deletion classification criteria are not always sufficient to eliminate the risk of misclassification of $p$ fhrp $2 / 3$ deletions. The use of advanced serological and next-generation sequencing methods improved the quality of our $p f h r p 2 / 3$ deletion assessment, allowed for a more robust evaluation of RDT performance, and enabled visualization of the genetic structure of the $p f h r p 2$ and $p f h r p 3$ genes and their flanking regions. While these methodologies are not widely available in resource-limited settings, they are now accessible through a network of laboratories that collaborate with the World Health Organization to support $p f h r p 2 / 3$ deletion surveillance ${ }^{47}$ and in select locales in sub-Saharan Africa with advanced laboratory capacity.

Symptomatic malaria due to non-falciparum species was common but usually occurred as part of mixed infections with $P$. falciparum. Although non-falciparum species are not detected by widely deployed HRP2based RDTs, co-infection with $P$. falciparum is expected to trigger a positive RDT result and prompt treatment with artemisinin-combination therapy according to current DRC guidelines. Therefore, complications due to untreated symptomatic, non-falciparum malaria are likely uncommon, although the risk of relapse by $P$. vivax or $P$. ovale without proper diagnosis and terminal prophylaxis remains. Our findings are generally in-line with prior reports of non-falciparum infection among asymptomatic subjects in the DRC ${ }^{48-50}$.

Strengths of this study include its geographically diverse sampling locations, robust pipeline of conventional and advanced laboratory methodologies, and relevance to malaria case management. Indeed, these findings directly informed the DRC national malaria control program's decision to continue the use of HRP2-based RDTs, despite evidence of $p f h r p 2$-negative parasites from our initial study of asymptomatic subjects. Our experience in the DRC confirms the importance of basing policy decisions on careful studies of the target population-individuals presenting to health facilities with symptomatic malaria-rather than convenience sampling determined by sample availability or access to study sites. 
Limitations include our inability to discriminate $p$ fhrp2/3-deleted from $p f h r p 2 / 3$-intact strains in individuals infected by multiple $P$. falciparum strains. Neither the conventional methods nor the advanced Luminex-based HRP2 antigenemia assessment and WGS methods employed here are well-suited to identify gene deletions in mixed infections. Indeed, assessment of whole-genome sequencing data confirmed mono-infection in only three of eight samples tested. We therefore cannot exclude the possibility that the five remaining samples included mixed infection that involved $p f h r p 2 / 3$-deleted strains. Recently developed multiplexed qPCR methods ${ }^{51}$ and amplicon-based deep sequencing approaches ${ }^{52}$ have potential to elucidate $p f h r p 2 / 3$-deleted minor variants in future large-scale surveys. Second, we restricted our $p$ fhrp $2 / 3$ deletion analysis to samples with $\geq 40$ parasites/ $\mu \mathrm{L}$. This requirement was necessary to reduce the risk of misclassification due to DNA concentrations below the pfhrp2/3 PCR assays' limits of detection ${ }^{13}$, but it prevents us from commenting on the prevalence of deletions in lower density infections. Third, enrollment occurred primarily in the rainy season, with only limited dry season enrollment near the end of the study in Bas-Uele. A modeling study predicts that $p f h r p 2 / 3$-deleted parasite prevalence may be underestimated during the rainy season, when individuals are more likely to be infected by multiple parasite strains ${ }^{53}$. Fourth, we could not evaluate associations between specific symptoms or temperature and RDT performance, as this data was only available for a subset of subjects. Finally, this study was restricted to three provinces. These provinces spanned a range of malaria prevalences, but they do not capture the full diversity of the DRC, which is Africa's second largest country by land mass and neighbors nine other countries.

In conclusion, ongoing HRP2-based RDT use is appropriate in the DRC. False-negative RDT results due to $p$ fhrp 2/3 deletions were not observed among symptomatic subjects. Most false-negative results in the DRC are likely due to low parasite densities, RDT failure, or operator error. Only a minority of non-falciparum malaria cases would be missed by an HRP2-based RDT testing strategy; co-infection with P. falciparum and non-falciparum species was common. Careful laboratory workflows are required during $p$ fhrp $2 / 3$ gene deletion analyses. Advanced serological and next-generation sequencing approaches can be used to improve the rigor and reproducibility of $p f h r p 2 / 3$ deletion surveillance efforts and to inform malaria diagnostic testing policy.

\section{Data availability}

Genomic sequencing data is available through the Sequence Read Archive (BioSample accession numbers: SAMN16711875-82). Datasets generated during the current study are not publicly available because they contain protected health information but are available from the authors upon reasonable request and with permission of SANRU.

Received: 27 August 2020; Accepted: 8 March 2021

Published online: 22 March 2021

\section{References}

1. World Health Organization. False-Negative RDT Results and Implications of New Reports of P. falciparum Histidine-Rich Protein 2/3 Gene Deletions (WHO, 2016).

2. Verma, A. K., Bharti, P. K. \& Das, A. HRP-2 deletion: A hole in the ship of malaria elimination. Lancet Infect. Dis. 18, 826-827 (2018).

3. Poti, K. E., Sullivan, D. J., Dondorp, A. M. \& Woodrow, C. J. HRP2: Transforming malaria diagnosis, but with caveats. Trends Parasitol. 36, 112-126 (2020).

4. Cheng, Q. et al. Plasmodium falciparum parasites lacking histidine-rich protein 2 and 3: A review and recommendations for accurate reporting. Malar. J. 13, 283 (2014).

5. Thomson, R. et al. Prevalence of Plasmodium falciparum lacking histidine-rich proteins 2 and 3: A systematic review. Bull. World Health Organ. 98, 558-568 (2020).

6. Watson, O. J. et al. Modelling the drivers of the spread of Plasmodium falciparum hrp2 gene deletions in sub-Saharan Africa. Elife https://doi.org/10.7554/eLife.25008 (2017).

7. Gatton, M. L. et al. Implications of parasites lacking Plasmodium falciparum histidine-rich protein 2 on malaria morbidity and control when rapid diagnostic tests are used for diagnosis. J. Infect. Dis. 215, 1156-1166 (2017).

8. Berhane, A. et al. Major threat to malaria control programs by Plasmodium falciparum lacking histidine-rich protein 2, Eritrea. Emerg. Infect. Dis. 24, 462-470 (2018).

9. Menegon, M. et al. Identification of Plasmodium falciparum isolates lacking histidine-rich protein 2 and 3 in Eritrea. Infect. Genet. Evol. 55, 131-134 (2017).

10. Parr, J. B. et al. Pfhrp2-deleted Plasmodium falciparum parasites in the Democratic Republic of the Congo: A national crosssectional survey. J. Infect. Dis. 216, 36-44 (2017).

11. Woodrow, C. J. \& Fanello, C. Pfhrp2 deletions in the Democratic Republic of Congo: Evidence of absence, or absence of evidence?. J. Infect. Dis. 216, 504-506 (2017).

12. Parr, J. B. \& Meshnick, S. R. Response to Woodrow and Fanello. J. Infect. Dis. 216, 503-504 (2017).

13. Parr, J. B., Anderson, O., Juliano, J. J. \& Meshnick, S. R. Streamlined, PCR-based testing for pfhrp2- and pfhrp3-negative Plasmodium falciparum. Malar. J. 17, 137 (2018).

14. Jones, S. et al. One-step PCR: A novel protocol for determination of pfhrp2 deletion status in Plasmodium falciparum. PLoS ONE 15, e0236369 (2020).

15. Watson, O. J. et al. False-negative malaria rapid diagnostic test results and their impact on community-based malaria surveys in sub-Saharan Africa. BMJ Glob. Health https://doi.org/10.1136/bmjgh-2019-001582 (2019).

16. Wu, L. et al. Comparison of diagnostics for the detection of asymptomatic Plasmodium falciparum infections to inform control and elimination strategies. Nature 528, S86-93 (2015).

17. President's Malaria Initiative. Democratic Republic of the Congo Malaria Operational Plan FY 2018. Available at https://www. pmi.gov/docs/default-source/default-document-library/malaria-operational-plans/fy-2018/fy-2018-democratic-republic-of-thecongo-malaria-operational-plan.pdf?sfvrsn $=5$.

18. Plowe, C. V., Djimde, A., Bouare, M., Doumbo, O. \& Wellems, T. E. Pyrimethamine and proguanil resistance-conferring mutations in Plasmodium falciparum dihydrofolate reductase: Polymerase chain reaction methods for surveillance in Africa. Am. J. Trop. Med. Hyg. 52, 565-568 (1995).

19. Pickard, A. L. et al. Resistance to antimalarials in southeast asia and genetic polymorphisms in pfmdr1. Antimicrob. Agents Chemother. 47, 2418-2423 (2003). 
20. Baker, J. et al. Genetic diversity of Plasmodium falciparum histidine-rich protein 2 (PfHRP2) and its effect on the performance of PfHRP2-based rapid diagnostic tests. J. Infect. Dis. 192, 870-877 (2005).

21. Koita, O. A. et al. False-negative rapid diagnostic tests for malaria and deletion of the histidine-rich repeat region of the hrp2 gene. Am. J. Trop. Med. Hyg. 86, 194-198 (2012).

22. Price, R. N. et al. Mefloquine resistance in Plasmodium falciparum and increased pfmdr1 gene copy number. Lancet 364, 438-447 (2004).

23. Afonina, I. et al. Primers with 5' flaps improve real-time PCR. Biotechniques 43, 770-774 (2007).

24. Morgan, A. P. et al. Falciparum malaria from coastal Tanzania and Zanzibar remains highly connected despite effective control efforts on the archipelago. Malar. J. 15, 47. https://doi.org/10.1186/s12936-020-3137-8 (2020).

25. Oyola, S. O. et al. Whole genome sequencing of Plasmodium falciparum from dried blood spots using selective whole genome amplification. Malar. J. 15, 597. https://doi.org/10.1186/s12936-016-1641-7 (2016).

26. Bolger, A. M., Lohse, M. \& Usadel, B. Trimmomatic: A flexible trimmer for Illumina sequence data. Bioinformatics 30, 2114-2120 (2014).

27. Li, H. Aligning sequence reads, clone sequences and assembly contigs with BWA-MEM. arXiv: 1303.3997 (2013).

28. Broad Institute. Picard tools. GitHub repository available at https://broadinstitute.github.io/picard/ (2016).

29. McKenna, A. et al. The genome analysis toolkit: A MapReduce framework for analyzing next-generation DNA sequencing data. Genome Res. 20, 1297-1303 (2010).

30. Quinlan, A. R. \& Hall, I. M. BEDTools: A flexible suite of utilities for comparing genomic features. Bioinformatics 26, 2 (2010).

31. Wickham, H. ggplot2: Elegant graphics for data analysis (Springer, Berlin, 2009).

32. Robinson, J. T. et al. Integrative genomics viewer. Nat. Biotechnol. 29, 24-26 (2011).

33. Auburn, S. et al. Characterization of within-host Plasmodium falciparum diversity using next-generation sequence data. PLoS ONE 7, e32891 (2012).

34. Morgan A. P. vcfdo. Available at: https://github.com/IDEELResearch/vcfdo (2019).

35. Plucinski, M. M. et al. Screening for Pfhrp2/3-deleted Plasmodium falciparum, non-falciparum, and low-density malaria infections by a multiplex antigen assay. J. Infect. Dis. 219, 437-447 (2019).

36. Kamau, E., Alemayehu, S., Feghali, K. C., Saunders, D. \& Ockenhouse, C. F. Multiplex qPCR for detection and absolute quantification of malaria. PLoS ONE 8, e71539 (2013).

37. Srisutham, S. et al. Four human Plasmodium species quantification using droplet digital PCR. PLoS ONE 12, e0175771 (2017).

38. Veron, V., Simon, S. \& Carme, B. Multiplex real-time PCR detection of P. falciparum, P. vivax and P. malariae in human blood samples. Exp. Parasitol. 121, 346-351 (2009).

39. Perandin, F. et al. Development of a real-time PCR assay for detection of Plasmodium falciparum, Plasmodium vivax, and Plasmodium ovale for routine clinical diagnosis. J. Clin. Microbiol. 42, 1214-1219 (2004).

40. Lucchi, N. W. et al. A new single-step PCR assay for the detection of the zoonotic malaria parasite Plasmodium knowlesi. PLoS ONE 7, e31848 (2012).

41. World Health Organization. Protocol for Estimating the Prevalence of pfhrp2/pfhrp3 Gene Deletions Among Symptomatic Falciparum Patients with False-Negative RDT Results (WHO, 2018).

42. Markwalter, C. F. et al. Characterization of Plasmodium lactate dehydrogenase and histidine-rich protein 2 clearance patterns via rapid on-bead detection from a single dried blood spot. Am. J. Trop. Med. Hyg. 98, 1389-1396 (2018).

43. Pati, P., Dhangadamajhi, G., Bal, M. \& Ranjit, M. High proportions of pfhrp2 gene deletion and performance of HRP2-based rapid diagnostic test in Plasmodium falciparum field isolates of Odisha. Malar. J. 17, 394 (2018).

44. Walker-Jonah, A., Dolan, S. A., Gwadz, R. W., Panton, L. J. \& Wellems, T. E. An RFLP map of the Plasmodium falciparum genome, recombination rates and favored linkage groups in a genetic cross. Mol. Biochem. Parasitol. 51, 313-320 (1992).

45. Sepúlveda, N. et al. Global analysis of Plasmodium falciparum histidine-rich protein-2 (pfhrp2) and pfhrp3 gene deletions using whole-genome sequencing data and meta-analysis. Infect. Genet. Evol. 62, 211-219 (2018).

46. Baker, J. et al. Global sequence variation in the histidine-rich proteins 2 and 3 of Plasmodium falciparum: Implications for the performance of malaria rapid diagnostic tests. Malar. J. 9, 2 (2010).

47. World Health Organization. Response Plan to pfhrp2 Gene Deletions (WHO, 2019).

48. Taylor, S. M. et al. Molecular malaria epidemiology: Mapping and burden estimates for the Democratic Republic of the Congo, 2007. PLoS ONE 6, e16420 (2011).

49. Brazeau, N. F. et al. The Epidemiology of Plasmodium vivax Among Adults in the Democratic Republic of the Congo: A NationallyRepresentative, Cross-Sectional Survey. Pre-print available on medRxiv at https://www.medrxiv.org/content/https://doi.org/10. 1101/2020.02.17.20024190v1.abstract. (2020).

50. Mitchell, C. L. et al. Under the radar: Epidemiology of Plasmodium ovale in the Democratic Republic of the Congo. J. Infect. Dis. https://doi.org/10.1093/infdis/jiaa478 (2020).

51. Grignard, L. et al. A novel multiplex qPCR assay for detection of Plasmodium falciparum with histidine-rich protein 2 and 3 (pfhrp2 and pfhrp3) deletions in polyclonal infections. EBioMedicine 55, 102757 (2020).

52. Verity, R. et al. The impact of antimalarial resistance on the genetic structure of Plasmodium falciparum in the DRC. Nat. Commun. 11, 2107 (2020).

53. Watson, O. J. et al. Impact of seasonal variations in Plasmodium falciparum malaria transmission on the surveillance of pfhrp2 gene deletions. Elife 8, 2 (2019).

\section{Acknowledgements}

The authors thank Steven Meshnick posthumously for his key role in conceptualizing this study and interpretation of results, Clark Cunningham and Kara Moser for assistance with variant calling and assessment of complexity of infection, and Nicholas Brazeau for assistance with visualization of genome coverage statistics. They also thank the study supervisors, staff, and participants. The following reagents were obtained through BEI Resources, NIAID, NIH: Genomic DNA from P. falciparum strain 3D7, MRA-102G, contributed by Daniel J. Carucci; $P$. falciparum strain HB3, MRA-155G, contributed by Thomas E. Wellems; P. falciparum strain Dd2, MRA-150G, contributed by David Walliker; and P. knowlesi strain H, MRA-456G, contributed by Alan W. Thomas; in addition to diagnostic plasmid containing the small subunit ribosomal RNA gene (18S) from Plasmodium vivax, MRA178, Plasmodium ovale, MRA-180, and Plasmodium malariae, MRA-179, contributed by Peter A. Zimmerman.

\section{Author contributions}

J.B.P., E.K., K.M., N.M., J.L.L., and A.N. conceptualized the study. E.K., F.P., P.M., K.M., N.M., A.L., J.A., S.U.E., J.W.O., P.M., J.L.L., E.M.S., A.K.T., A.N., A.K. supervised and performed field work. J.B.P., K.L.T., C.E.M., M.D., A.P., J.J.J., and E.R. performed laboratory analyses. J.B.P. prepared the figures and wrote the first draft. All authors reviewed and approved the final draft. 


\section{Funding}

This work was supported by the Global Fund to Fight AIDS, Tuberculosis, and Malaria. It was also partially supported by awards from the National Institutes of Allergy and Infectious Diseases [R01AI132547 to JBP and JJJ, R21AI148579 to JBP], the National Institute of General Medical Sciences [T32GM008719 to CEM], and the Doris Duke Charitable Foundation to JBP.

\section{Competing interests}

JBP reports research support from the World Health Organization related to the scope of the present study and non-financial support from Abbott Laboratories, outside the scope of the present study. All other authors declare no competing interests.

\section{Additional information}

Supplementary Information The online version contains supplementary material available at https://doi.org/ 10.1038/s41598-021-85913-z.

Correspondence and requests for materials should be addressed to J.B.P.

Reprints and permissions information is available at www.nature.com/reprints.

Publisher's note Springer Nature remains neutral with regard to jurisdictional claims in published maps and institutional affiliations.

(c) (i) Open Access This article is licensed under a Creative Commons Attribution 4.0 International License, which permits use, sharing, adaptation, distribution and reproduction in any medium or format, as long as you give appropriate credit to the original author(s) and the source, provide a link to the Creative Commons licence, and indicate if changes were made. The images or other third party material in this article are included in the article's Creative Commons licence, unless indicated otherwise in a credit line to the material. If material is not included in the article's Creative Commons licence and your intended use is not permitted by statutory regulation or exceeds the permitted use, you will need to obtain permission directly from the copyright holder. To view a copy of this licence, visit http://creativecommons.org/licenses/by/4.0/.

(C) The Author(s) 2021 